\title{
Anthropometric, Physical, Cardiorespiratory Fitness and Lipids and Lipoproteins Profile of Young Indian Children of 10-16 Years Age Group
}

\author{
I. Manna ${ }^{1, *}$, S. R. Pan ${ }^{2}$, M. Chowdhury ${ }^{3}$ \\ ${ }^{1}$ Department of Physiology, Midnapore College, Midnapore, West Bengal, India \\ ${ }^{2}$ Department of Physical Education, Jhargram Raj College, Jhargram, West Bengal, India \\ ${ }^{3}$ Department of Community Medicine, B.S. Medical College, Bankura, West Bengal, India \\ *Corresponding author: indranil_manna@yahoo.com
}

Received June 16, 2014; Revised June 26, 2014; Accepted July 02, 2014

\begin{abstract}
Sports talent may be identified from young children when they show interest in different sports. Anthropometric, physical, cardiorespiratory fitness and lipids profiles contribute to selection procedures. The present study was undertaken to investigate the anthropometric, physical, cardiorespiratiory fitness and lipids and lipoprotein profiles of 10-16 yrs children, in order to identify sports talent in them. A total of 225 male children of 10-16 yrs age volunteered for this study; were divided equally into 3 groups (i) Prepubertal (age-11.0 $\pm 0.8 y r s, n=75$ ); (ii) Pubertal (age $13.5 \pm 0.5$ yrs, n=75); (iii) Postpubertal (age $15.5 \pm 0.5$ yrs, $n=75$ ). Selected anthropometric, physical and cardiorespiratiory fitness variables were measured for each group. A significantly $(\mathrm{P}<0.05)$ greater height, body mass, BSA, LBM, mid upper arm circumference, hip and trunk flexibility, grip strengths, abdominal strength, elastic leg strength, maximum speed, peak power, $\mathrm{VO}_{2 \max }$, FVC, FEV1, PEFR, blood pressure and serum HDL-C level were observed in Postpubertal children when compared to Prepubertal and Pubertal children. However, a significantly $(\mathrm{P}<0.05)$ lower percent body fat, reaction time, maximal heart rate, recovery heart rates and serum triglyceride level were noted in Postpubertal children when compared to Prepubertal and Pubertal children. The waist- hip ratio of pubertal children was noted significantly higher $(\mathrm{P}<0.05)$ when compared to prepubertal and postpurbertal children. No significant change was reported in BMI, resting heart rate, serum total cholesterol and LDL-C levels among the groups. Identification of children at early stage of their growth and development may produce elite athletes in the future. Talent identification also can be used as a counseling technique that helps to discover and explore areas of talent for particular athletes.
\end{abstract}

Keywords: body composition, $\mathrm{VO}_{2 \max }$, power, strength, lipid profile

Cite This Article: I. Manna, S. R. Pan, and M. Chowdhury, "Anthropometric, Physical, Cardiorespiratory Fitness and Lipids and Lipoproteins Profile of Young Indian Children of 10-16 Years Age Group.” American Journal of Sports Science and Medicine, vol. 2, no. 4 (2014): 154-160. doi: 10.12691/ajssm-2-4-7.

\section{Introduction}

Sports talent may be identified from the school children 10-16 yrs age group when they show interest in different sports [1]. Anthropometric, physical and cardiorespiratory fitness profiles contribute to selection procedures in different sports events [2]. Besides success in track and field discipline is based on the synthesis of anthropometric characteristics and motor abilities as well as optimal technique [3]. But overall characteristics are also influenced by genetic inheritance, morphology, personal interest and habitual activity. Cardiorespiratory fitness variables such as maximal aerobic capacity $\left(\mathrm{VO}_{2 \max }\right)$, heart rate, blood pressure and pulmonary functions reflect the overall capacity of the cardiovascular and respiratory systems and the ability to carry out prolonged exercise [4]. Hence, Cardiorespiratory fitness has been considered as a direct measure of the physiological status of the individual
$[4,5]$. The gold standard for the measurement of cardiorespiratory fitness is the maximal oxygen uptake $\left(\mathrm{VO}_{2 \max }\right)$. The level of cardiorespiratory fitness is highly associated with the performance of other health-related fitness parameters such as strength and power output in young people and in adults [6]. Lipids have important beneficial biological functions. These include usage of triglycerides for energy production, fat storage in adipose tissues, and usage of cholesterol as a component in phospholipids of cellular membranes or in the synthesis of steroid hormones $[7,8]$. Elevated plasma cholesterol concentrations have been implicated in the development of coronary artery disease (CAD) [7,9]. Regular monitoring of these health variables of sports children can provide valuable information about their health, metabolic and cardiovascular status.

To identify athletic potentiality, norms of the anthropometric, physical, cardiorespiratory fitness and lipids profiles have an importance because they represent the health, metabolic and cardiovascular status of the 
athletes, which relates with the achievement level of a particular group [10]. Various factors like socio-economic condition, diet, physical activity may reflect on these variables. Thus there is a wide range of normalcy and the need to develop local norms has been emphasized. Several studies have been carried out on the physical [6] and cardiorespiratory [5] fitness status of the children of school-age populations. In India, limited studies on the anthropometric, physical [11,12,13], cardiorespiratiory fitness [12,13] and lipids and lipoproteins profiles $[12,13,14]$ of children have been reported. In view of the above, a study was undertaken to investigate the anthropometric, physical, cardiorespiratiory fitness and lipids and lipoprotein profiles of young Indian children of 10-16 years age group in order to identify potentiality and sports talent in them.

\section{Methods}

\subsection{Subjects}

A total of 225 male children of 10-16 yrs age volunteered for this study. The children were selected after proper medical checkups from West Midnapore districts of West Bengal, India. The subjects were equally divided into 3 groups (i) Prepubertal (10-12 yrs age; age$11.0 \pm 0.8 y r s, n=75)$; (ii) Pubertal (13-14 yrs age; age 13.5 \pm 0.5 yrs, $n=75$ ); (iii) Postpubertal (15-16 yrs age; age $15.5 \pm 0.5$ yrs, $n=75$ ). The subjects were informed about the possible complications of the study and gave their consent. Parental consent was also taken from the participants of this study. The institutional review board and ethical board approval was also obtained for the present study.

\subsection{Measurement of Anthropometric Variables}

Height and body mass were measured using standard methodology [15]. Body mass index (BMI) and Body surface area (BSA) were derived from the height and body mass using standard equations [15]. Measurements of hips and waist of the subject was taken by a steel tape using standard procedure, and the waist- hip ratio (WHR) was determined by standard equation [15]. Mid upper arm circumference (MUAC) of the subject was taken by a steel tape using standard procedure [15]. A skin fold calliper (Mitutoyo, Japan) was used to assess the body fat percentage, from biceps, triceps, sub scapular and suprailiac sites. Body density was calculated according to the formulae of Durnin and Womersley [16]. Body fat was derived using the standard equation of Siri [17]. Subsequently, lean body mass (LBM) was derived by subtracting fat mass from total body mass using the standard equation [15].

\subsection{Assessment of Physical Fitness}

Reaction time of the subject was assessed by ruler drop test using standard procedure [15]. Modified sit and reach test (MSRT) was applied using standard procedure in order to assess subject's hip and trunk flexibility [15]. Sit ups test (SUT) was performed using standard procedure to monitor the development of the subject's abdominal strength ${ }^{[15]}$. Standing long jump test (SLJT) was performed to monitor the development of the subject's elastic leg strength [15]. A grip strength dynamometer (T.K.K.5001 Grip-A, Japan) was used to record the strength of grip muscles of both hands following a standard methodology [15]. To monitor the development of the subject's ability to effectively and efficiently build up acceleration from a standing start to maximum speed, 30 meter acceleration test (30MAT) was performed using standard procedure [15]. Margaria Kalamen Power Test was used to monitor subject's peak power using the standard procedure [18].

\subsection{Bottom of Form}

\subsubsection{Assessment of Cardiorespiratory fitness:}

Subject was asked to take rest for $15 \mathrm{~min}$ and the heart rate and blood pressure were recorded. Maximal heart rate (HRmax) and recovery heart rates were recorded following a maximal exhaustive exercise. Maximal aerobic capacity $\left(\mathrm{VO}_{2 \max }\right)$ was measured indirectly using Queen's College step test following standard procedure [19]. To assessment of lung functions of the subjects forced vital capacity (FVC), forced expiratory volume in 1 second (FEV1) and peak expiratory flow rate (PEFR) were recorded using an electronic spirometer (Micro I Spirometer, CareFusion, UK) following a standard procedure [20].

\subsubsection{Assessment of Lipid Profiles}

A $5 \mathrm{ml}$ of venous blood was drawn from an antecubital vein after a 12-hours fast and 24 hours after the last bout of exercise for the subsequent determination of selected biochemical parameters. The biochemical parameters were measured using standard methodology. All the reagents were supplied from Boehringer Mannhein, USA. Serum triglycerides [21], serum total cholesterol (TC) [22] and high-density lipoprotein cholesterol (HDL-C) [22] were determined by enzymatic method. Low-density lipoprotein cholesterol (LDL-C) was indirectly assessed following standard equation [23].

\subsection{Statistical Analysis}

All the values of anthropometric, physical, cardirespiratiory fitness and lipids and lipoproteins profiles were expressed as mean and standard deviation (SD). Analysis of Variance (ANOVA) followed by multiple comparison tests was performed to find out the significant difference in selected anthropometric, physical, cardirespiratiory fitness and lipids and lipoproteins profiles among the groups. In each case the significant level was chosen at 0.05 levels. Accordingly, a statistical software package (SPSS) was used.

\section{Results}

Anthropometric parameters showed variations among the Prepubertal, Pubertal and Postpubertal children. A significantly $(\mathrm{P}<0.05)$ higher height, body mass, body surface area (BSA), lean body mass (LBM) and mid upper arm circumference (MUAC) were observed in Postpubertal children when compared to Prepubertal and 
Pubertal children. On the other hand, a significantly $(\mathrm{P}<0.05)$ lower percent body fat was noted in Postpubertal children when compared to Prepubertal and Pubertal children. The waist- hip ratio (WHR) of pubertal children was noted significantly higher $(\mathrm{P}<0.05)$ when compared to prepubertal and postpurbertal children. However, no significant change was reported in body mass index (BMI) and total body fat among the groups (Table 1).

Table 1. Anthropometric variable of 10-16 years Children

\begin{tabular}{|c|c|c|c|}
\hline Variables & $\begin{array}{c}\text { Prepubertal } \\
(10-12 \text { yrs })\end{array}$ & $\begin{array}{c}\text { Pubertal } \\
(13-14 \text { yrs })\end{array}$ & $\begin{array}{c}\text { Postpubertal } \\
(15-16 \text { yrs })\end{array}$ \\
\hline Height (cm) & $141.1 \pm 3.6$ & $154.1 \pm 3.5^{*}$ & $162.5 \pm 3.7^{* \#}$ \\
\hline Body mass (kg) & $35.2 \pm 4.5$ & $42.6 \pm 6.3^{*}$ & $47.4 \pm 5.2^{* \#}$ \\
\hline BMI & $17.4 \pm 1.6$ & $18.1 \pm 1.7^{\mathrm{NS}}$ & $18.1 \pm 1.4^{\mathrm{NS}}$ \\
\hline BSA & $1.2 \pm 0.05$ & $1.3 \pm 0.06^{*}$ & $1.5 \pm 0.05^{\#}$ \\
\hline Body fat (\%) & $19.5 \pm 3.5$ & $16.7 \pm 4.2^{*}$ & $14.3 \pm 4.1^{* \#}$ \\
\hline Body Fat (kg) & $6.5 \pm 1.6$ & $7.1 \pm 1.4^{\mathrm{NS}}$ & $6.9 \pm 1.8^{\mathrm{NS}}$ \\
\hline LBM (kg) & $29.2 \pm 3.5$ & $35.4 \pm 5.7^{*}$ & $40.5 \pm 4.1^{* \#}$ \\
\hline WHR & $0.8 \pm 0.03$ & $0.9 \pm 0.03^{*}$ & $0.8 \pm 0.01^{\#}$ \\
\hline MUAC (cm) & $18.6 \pm 2.5$ & $21.1 \pm 2.3^{*}$ & $24.3 \pm 2.7^{* \#}$ \\
\hline
\end{tabular}

All the values were expressed as mean and standard deviation (SD), $\mathrm{n}=75$; ANOVA followed by multiple comparison tests; ${ }^{*} \mathrm{P}<0.05$ when compare to prepubertal age group, $\# \mathrm{P}<0.05$, when compare to pubertal age group, $\mathrm{NS}=$ not significant; $\mathrm{BMI}=$ body mass index, $\mathrm{BSA}=$ body surface area, LBM= lean body mass, WHR= waist- hip ratio, MUAC= mid upper arm circumference.

Physical fitness variables showed remarkable differences among the Prepubertal, Pubertal and Postpubertal children. A significantly $(\mathrm{P}<0.05)$ higher hip and trunk flexibility as measured by modified sit and reach test (MSRT) score, abdominal strength as measured by sit ups test (SUT) score, elastic leg strength as measured by standing long jump test (SLJT) score, grip strengths of both hands, maximum speed as measured by lower 30 meter acceleration test (30MAT) score, and peak power output were observed in Postpubertal children when compared to Prepubertal and Pubertal children. On the other hand, a significantly $(\mathrm{P}<0.05)$ lower reaction time as measured by ruler drop test (RDT) was noted in Postpubertal children when compared to Prepubertal and Pubertal children (Table 2).

Table 2. Physical fitness variable of 10-16 years Children

\begin{tabular}{|c|c|c|c|}
\hline Variables & $\begin{array}{c}\text { Prepubertal } \\
(10-12 \text { yrs })\end{array}$ & $\begin{array}{c}\text { Pubertal } \\
(13-14 \text { yrs })\end{array}$ & $\begin{array}{c}\text { Postpubertal } \\
(15-16 \mathrm{yrs})\end{array}$ \\
\hline RDT (cm) & $18.3 \pm 2.7$ & $16.1 \pm 2.5^{*}$ & $14.1 \pm 2.6^{* \#}$ \\
\hline MSRT (cm) & $11.5 \pm 1.9$ & $15.3 \pm 1.6^{*}$ & $17.2 \pm 1.4^{* \#}$ \\
\hline SUT (in 30 sec) & $16.2 \pm 1.4$ & $17.8 \pm 1.7^{*}$ & $20.2 \pm 1.5^{* \#}$ \\
\hline SLJT (m) & $1.6 \pm 0.7$ & $1.8 \pm 0.5^{*}$ & $2.5 \pm 0.4^{* \#}$ \\
\hline GSTR (kg) & $18.7 \pm 3.1$ & $25.1 \pm 3.6^{*}$ & $32.7 \pm 3.3^{* \#}$ \\
\hline GSTL (kg) & $18.1 \pm 2.4$ & $24.5 \pm 3.8^{*}$ & $31.5 \pm 3.4^{* \#}$ \\
\hline 30MAT (sec) & $6.1 \pm 0.7$ & $5.5 \pm 0.5^{*}$ & $4.9 \pm 0.4^{* \#}$ \\
\hline Peak power (watt) & $509.6 \pm 217.5$ & $721.3 \pm 17.3^{*}$ & $834.7 \pm 14.6^{* \#}$ \\
\hline
\end{tabular}

All the values were expressed as mean and standard deviation (SD), $\mathrm{n}=75$; ANOVA followed by multiple comparison tests; ${ }^{*} \mathrm{P}<0.05$ when compare to prepubertal age group, $\# \mathrm{P}<0.05$, when compare to pubertal age group; RDT = ruler drop test, MSRT= modified sit and reach test, SUT = sit ups test, SLJT= standing long jump test, GSTR= grip strength of right hand, GSTL= grip strength of left hand, 30MAT= 30 meter acceleration test.

Cardiorespiratory fitness variables showed remarkable changes among the Prepubertal, Pubertal and Postpubertal children. A significantly $(\mathrm{P}<0.05)$ higher $\mathrm{VO}_{2 \max }, \mathrm{FVC}$, FEV1, PEFR, resting systolic and diastolic blood pressure were observed in Postpubertal children when compared to Prepubertal and Pubertal children. On the other hand, a significantly $(\mathrm{P}<0.05)$ lower maximal heart rate (HRmax) and recovery heart rates were noted in Postpubertal children when compared to Prepubertal and Pubertal children. However, no significant change was reported in resting heart rate among the groups (Table 3).

Table 3. Cardiorespiratory variable of 10-16 years Children

\begin{tabular}{|c|c|c|c|}
\hline Variables & $\begin{array}{c}\text { Prepubertal } \\
(10-12 \text { yrs })\end{array}$ & $\begin{array}{c}\text { Pubertal } \\
(13-14 \text { yrs })\end{array}$ & $\begin{array}{c}\text { Postpubertal } \\
(15-16 \text { yrs })\end{array}$ \\
\hline RHR (beats/min) & $63.5 \pm 3.1$ & $64.1 \pm 3.3 \mathrm{NS}$ & $62.4 \pm 2.6 \mathrm{NS}$ \\
\hline HRmax (beats/min) & $193.8 \pm 7.1$ & $191.5 \pm 4.9^{*}$ & $184.6 \pm 5.7^{*} \#$ \\
\hline RecHR1 (beats/min) & $156.4 \pm 5.2$ & $147.6 \pm 6.4^{*}$ & $141.5 \pm 4.3^{*} \#$ \\
\hline RecHR2 (beats/min) & $133.7 \pm 4.2$ & $126.4 \pm 3.4^{*}$ & $119.8 \pm 4.2^{* \#}$ \\
\hline RecHR3 (beats/min) & $114.5 \pm 3.7$ & $107.6 \pm 3.4^{*}$ & $101.7 \pm 3.8^{* \#}$ \\
\hline RSBP (mmHg) & $97.4 \pm 5.2$ & $100.4 \pm 5.1^{*}$ & $108.7 \pm 6.5^{*} \#$ \\
\hline RDBP (mmHg) & $66.8 \pm 4.6$ & $67.2 \pm 4.3^{*}$ & $70.4 \pm 4.5^{*} \#$ \\
\hline $\begin{array}{c}\text { VO2max } \\
\text { (ml/kg/min) }\end{array}$ & $38.3 \pm 3.1$ & $43.9 \pm 3.4^{*}$ & $46.2 \pm 3.6^{* \#}$ \\
\hline FVC (l) & $1.9 \pm 0.2$ & $2.3 \pm 0.2 \mathrm{NS}$ & $2.7 \pm 0.1^{* \#}$ \\
\hline FEV1 (l) & $1.7 \pm 0.1$ & $2.1 \pm 0.1^{*}$ & $2.5 \pm 0.1^{* \#}$ \\
\hline PEFR (l) & $220.1 \pm 12.4$ & $291.7 \pm$ & $349.3 \pm 13.5^{* \#}$ \\
\hline
\end{tabular}

All the values were expressed as mean and standard deviation (SD), $\mathrm{n}=75$; ANOVA followed by multiple comparison tests; ${ }^{*} \mathrm{P}<0.05$ when compare to prepubertal age group, $\# \mathrm{P}<0.05$, when compare to pubertal age group, NS= not significant; RHR= resting heart rate, HRmax= maximal heart rate, RecHR1 $=$ recovery heart rate in $1^{\text {st }}$ min, RecHR2= recovery heart rate in $2^{\text {nd }} \mathrm{min}$, RecHR3= recovery heart rate in $3^{\text {rd }} \mathrm{min}$, $\mathrm{RSBP}=$ resting systolic blood pressure, $\mathrm{RDBP}=$ resting diastolic blood pressure, $\mathrm{VO} 2 \mathrm{max}=$ maximal aerobic capacity, $\mathrm{FVC}=$ forced vital capacity, FEV1=forced expiratory volume in 1 second, PEFR= peak expiratory flow rate.

Lipids and lipoprotein profile represents the health and metabolic status of the athletes. A significantly lower $(\mathrm{P}<0.05)$ serum triglyceride level was noted in Postpubertal children when compared to Prepubertal and Pubertal children. On the other hand, higher $(\mathrm{P}<0.05)$ serum HDL-C level was noted in Pubertal and Postpubertal children when compared to Prepubertal children. However, no significant change was noted in serum total cholesterol and LDL-C levels among the Prepubertal, Pubertal and Postpubertal children (Table 4).

Table 4. Lipids and lipoproteins profiles of 10-16 years Children

\begin{tabular}{|c|c|c|c|}
\hline Variables & $\begin{array}{c}\text { Prepubertal } \\
(10-12 \text { yrs })\end{array}$ & $\begin{array}{c}\text { Pubertal } \\
(13-14 \mathrm{yrs})\end{array}$ & $\begin{array}{c}\text { Postpubertal } \\
(15-16 \mathrm{yrs})\end{array}$ \\
\hline TC $(\mathrm{mg} / \mathrm{dl})$ & $151.2 \pm 11.5$ & $146.4 \pm 12.1$ & $149.3 \pm 10.5$ \\
\hline TG $(\mathrm{mg} / \mathrm{dl})$ & $84.0 \pm 9.8$ & $78.7 \pm 8.4$ & $74.5^{*} \pm 9.9$ \\
\hline HDL-C (mg/dl) & $41.6 \pm 5.0$ & $42.7^{*} \pm 6.9$ & $46.9^{*} \pm 6.6$ \\
\hline LDL-C (mg/dl) & $90.1 \pm 8.2$ & $89.7 \pm 8.6$ & $89.0 \pm 7.0$ \\
\hline
\end{tabular}

All the values were expressed as mean and standard deviation (SD), $\mathrm{n}=75$; ANOVA followed by multiple comparison tests; $* \mathrm{P}<0.05$ when compare to prepubertal age group, $\# \mathrm{P}<0.05$, when compare to pubertal age group, NS= not significant; serum total cholesterol (TC), Serum triglycerides (TG), high-density lipoprotein cholesterol (HDL-C), Lowdensity lipoprotein cholesterol (LDL-C).

\section{Discussion}

Childhood and adolescence are crucial periods of life, since dramatic physiological and psychological changes take place at these ages. Physical growth in children is measured by changes in body size and/or composition as well as physical profile [4]. During childhood and adolescence, body size and composition markedly change. These changes are strongly associated with the development of various physical performance characteristics. At the same time, anthropometry and body composition during adolescence are predictors of risk factors for cardiovascular disease, diabetes, and many types of chronic diseases [24,25] which occur in adults $[26,27,28]$. Hence, determining anthropometry and body composition during childhood and adolescence would be 
of interest to those working in both sports sciences and medicine. Body size (height, body mass, BMI and BSA) play important role during selection of players [29,30,31]. The tall players are recruited as in athletics, soccer, volleyball and other games. Although, game like filed hockey has no significant impact on height; however a standard height should be maintained for selection of players for each sports discipline. Body mass is a considerable factor in games and sports, since body contact is essential in like soccer, field hockey and some other games [32,33]. Body mass index (BMI) has been used as a simple anthropometric index which reflects the current nutritional status of an individual, and that of body surface area (BSA) can be made of an individual's daily resting energy expenditure [4]. In the present study, a significantly higher height, body mass and BSA were observed in Postpubertal children when compared to Prepubertal and Pubertal children. However, no significant change was reported in BMI among the groups. The possible reason for the increase in height Prepubertal to Pubertal and Postpubertal children might be the osteotropic response to exercise. The osteotropic effect of exercise is dependent on load dynamics, the volume, intensity and duration of training, administered on the individual and the period in life when exposure occurs [34]. In addition, the hormonal regulation of skeleton is unique in each stage of life [35]. The gain in height is dependent on growth hormone and exercise is a potent stimulus for growth hormone [35]. It has been reported that genetic influence can alter morphological status only within a narrow limit, set by his genotype [36]. Growth in body weight follows the same trend as in case of height. Increment in body weight in each age category may be due to the increment in bone and muscle weight. Increase in muscle mass with age, appears to result primarily from hypertrophy of existing fibres. The gain in weight is dependent on growth hormone and exercise is a potent stimulus for growth hormone [35]. Apart from the hormonal effects the neural maturity also helps to gain desirable body weight in the athletes [37]. It is possible that a particular body size will encourage acquisition of certain skills and force gravitation towards a specific playing position: this is likely to occur before maturity so that the individual will tend to favour one positional role before playing at senior level [32]. The waist to hip ratio (WHR) has been shown to be related to the risk of coronary heart disease $[38,39,40]$. Mid-upper arm circumference (MUAC) is a measure of nutritional status $[38,41]$. The waist- hip ratio (WHR) of pubertal children was noted significantly higher $(\mathrm{P}<0.05)$ when compared to prepubertal and postpurbertal children. Moreover, a significantly higher mid upper arm circumference (MUAC) was observed in Postpubertal children when compared to Prepubertal and Pubertal children. The changes might be because of level of maturation factors and / or motivation, and exposure to long term and higher intensity of training among the Postpubertal children when compared with Prepubertal and Pubertal children. Similar findings were also noted by other research groups who reported significant change in these parameters with the advancement of age, level of maturation and exposure to high intensity of exercise for long time among the children [42,43,44]. Monitoring of anthropometry and body composition at regular intervals is essential for selection of athletes for competitions. In addition, the anthropometric variables can predict the risk of obesity, cardiovascular and other diseases.

The percentage of body fat plays an important role for the assessment of physical fitness of the players $[32,45,46]$. Generally, the amount of fat in an adult male in his mid-twenties is about $16.5 \%$ of body weight $[4,37,47]$. A lean body is desirable for all sports discipline $[32,48,49]$. A low-body fat may improve athletic performance by improving the strength-to-weight ratio $[4,37,47]$. Excess body fat adds to the load without contributing to the body's force-producing capacity $[4,37,47]$. A significantly lower $(P<0.05)$ percent body fat was observed in Postpubertal children when compared to Prepubertal and Pubertal children. The lower body fat values in the postpubertal children might be because of exposure to long term and higher intensity of aerobic endurance training when compared with Prepubertal and Pubertal children. However, significant increase in LBM was noted in Postpubertal children when compared to Prepubertal and Pubertal children. This might be again due to long term effect of exercise among the Postpubertal children than Prepubertal and Pubertal children which reduces the body fat and which shows higher LBM among the Postpubertal children $[31,47,48]$. It can be stated that excess body fat can limit the aerobic and anaerobic performance of the players. Similar observations have been noted by other research groups $[31,47,48]$. The observations of our study may be supported by several studies, where decrease in body fat was noted with the advancement of age of the players [31,47]. Therefore, monitoring of body composition at regular intervals is essential for selection of athletes for competitions and during the training seasons.

Physical fitness of the athletes can be assessed by measuring motor skills and activities such as reaction time, hip and trunk flexibility, abdominal strength, elastic leg strength, grip strengths, maximum speed and peak power output. The reaction time is the time the athletes take for the body to react to a stimulus. The reaction time is very important for the track and field athletes as well as for players of different sports disciplines $[1,4]$. In the present study, a significantly $(\mathrm{P}<0.05)$ lower reaction time as measured by ruler drop test (RDT) was noted in Postpubertal children when compared to Prepubertal and Pubertal children. Flexibility is the ability to move a joint or series of joints smoothly and easily throughout a full range of motion. An athlete who has a restricted range of motion will realize a decrease in performance capabilities. Flexibility is important in preventing injury to the musculotendinous and skeletal anatomy $[1,4]$. There are some factors that limit flexibility are bony structure, excessive fat, skin, muscles and tendons, and connective tissues. With the exception of bony structure, age, and gender, all of the other factors that limit flexibility may be altered to increase range of joint motion. In the present study, a significantly $(\mathrm{P}<0.05)$ higher hip and trunk flexibility as measured by modified sit and reach test (MSRT) score was observed in Postpubertal children when compared to Prepubertal and Pubertal children. Strength is the central component of a athletics training program particularly for short distance run, through events, jump events and in different games [32,46,47]. Abdominal strength is important to monitor the development of the 
athlete's muscular endurance. To elastic leg strength is important to monitor the development of the athlete's muscular power. On the other hand, strength of grip muscle also has significant impacts on the performance of athletes, which is needed for throw-in, catching, serving, smashing or fisting the ball $[32,47]$. In the present study, a significantly $(\mathrm{P}<0.05)$ higher abdominal strength as measured by sit ups test (SUT) score, elastic leg strength as measured by standing long jump test (SLJT) score and grip strengths of both hands were observed in Postpubertal children when compared to Prepubertal and Pubertal children. Assessment of speed is important for selection of athletes in teams. Speed relates to the ability to perform a movement within a short time period. Power is the amount of work done or energy transferred per unit of time. Muscular power is the ability to use strength quickly to produce an explosive effort. Sports like short distance run, soccer, field hockey etc. demands high seep and power output as quick acceleration and deceleration are important in this sport [32,47]. Repeated back-to-back sprints make speed and tolerance to lactic acid an important characteristic in athletes [32,47]. A high speed and power output are essential for such activities [32,47]. Thus a high speed and power output helps to develop sprint quality of the athletes [32,47]. In the present study, a significantly $(\mathrm{P}<0.05)$ higher maximum speed as measured by lower 30 meter acceleration test (30MAT) score, and peak power output were observed in Postpubertal children when compared to Prepubertal and Pubertal children. The lower reaction time; and higher level of flexibility, abdominal strength, elastic leg strength and grip strength, speed and power of the postpubertal children might be because of exposure to long term and higher intensity of training when compared with Prepubertal and Pubertal children. Moreover, this might be because of level of maturation factors and / or motivation of the Postpubertal children when compared with Prepubertal and Pubertal children. Similar findings were also noted by other research groups who reported significant reduction of reaction time; and elevation in flexibility, abdominal strength, elastic leg strength and grip strength, speed and power with the advancement of age, level of maturation and exposure to high intensity of exercise for long time among the children [31,42,43,44,47]. Monitoring of the motor skills and activities such as reaction time, hip and trunk flexibility, abdominal strength, elastic leg strength, grip strengths, maximum speed and peak power output at regular intervals is essential for selection of athletes for competitions and during the training seasons.

Heart rate and blood pressure are essential for assessing cardiovascular fitness of the athletes. Heart rate increases with an increase in work intensity and shows a linear relationship with work rate [49]. The highest rate at which the heart can beat is the maximal heart rate (HRmax). Quick recovery from strenuous exercise is important in sports which involve intermittent efforts interspersed with short rests $[4,37,50]$. The heart rate recovery curve is an excellent tool for tracking a person's progress during a training program $[4,37]$. A significantly $(\mathrm{P}<0.05)$ lower maximal heart rate (HRmax) and recovery heart rates were noted in Postpubertal children when compared to Prepubertal and Pubertal children. However, no significant change was reported in resting heart rate among the groups. On the other hand, a significantly $(\mathrm{P}<0.05)$ higher resting systolic and diastolic blood pressure were observed in Postpubertal children when compared to Prepubertal and Pubertal children. Exercise cardio acceleration results from release of parasympathetic inhibition at low exercise intensities and from both parasympathetic inhibition and sympathetic activation at moderate intensities [4,37]. Nevertheless, parasympathetic activation is considered to be the main mechanism underlying exponential cardio deceleration after exercise [4,37]. The results of the present study suggest that the strain on the circulatory system during sports activities is relatively high. Exercising at this intensity should provide a good training stimulus. Therefore, heart rate and blood pressure monitoring is essential for selection of athletes and during the training seasons.

The maximal oxygen uptake $\left(\mathrm{VO}_{2 \max }\right)$ is the best overall measure of aerobic power [44,51]. Aerobic capacity certainly plays an important role in athletics activities and has a major influence on technical performance and tactical choices $[4,37,47]$. A significantly $(\mathrm{P}<0.05)$ higher $\mathrm{VO}_{2 \max }$ was observed in Postpubertal children when compared to Prepubertal and Pubertal children. The higher level of $\mathrm{VO}_{2 \max }$ value in the postpubertal children may be because of exposure to long term and higher intensity of aerobic endurance training compared with Prepubertal and Pubertal children. The increase in $\mathrm{VO}_{2 \max }$ might be due to an increase in the systemic a-v O2 difference and stroke volume [4,37]. Moreover, these changes might be the result of increased volume of endurance training [4,37]. The aerobic endurance training enhances the activity of the cardiovascular system as well as developed oxidative capacity of the skeletal muscles which leads to an increase in the delivery of oxygen to working muscles [4,37]. This is accepted as the main reason for elevation of $\mathrm{VO}_{2 \max }[4$, 30]. Similar observation has been reported previously $[32,47,52]$. The extent by which $\mathrm{VO}_{2 \max }$ could be changed with training also depends on the starting point [4,37]. The fitter an individual is to begin with, the less potential there is for an increase and most elite athletes hit this peak early in their career $[4,37]$. There also seems to be a genetic upper limit beyond which further increases in either intensity or volume have no effect on aerobic power [4,37]. Other than tactical and technical aspects of soccer, monitoring of $\mathrm{VO}_{2 \max }$ is essential during the training phases, which helps the coaches for selection of players for training and competition.

Lung function tests are of little value for predicting fitness and exercise performance, provided that the values fall within a normal range. The values of FVC, FEV1 and PEFR are used as indicators of lung disease [4,37]. In the present study, a significantly $(\mathrm{P}<0.05)$ higher FVC, FEV1 and PEFR were observed in Postpubertal children when compared to Prepubertal and Pubertal children. The higher FVC, FEV1 and PEFR of the postpubertal children might be because of exposure to long term and higher intensity of aerobic endurance training when compared with Prepubertal and Pubertal children. Moreover, this might be because of level of maturation factors and / or motivation of the Postpubertal children when compared with Prepubertal and Pubertal children. Monitoring of lung function tests like FVC, FEV1 and PEFR at regular intervals may provide information about the respiratory 
status of the athletes. In addition, these values are essential for selection of athletes for the training and competitions.

Lipids and lipoprotein profiles indicate the cardiovascular and metabolic status of athletes. Activity levels have significant impacts on the lipids and lipoprotein levels of athletes [7]. As the performance level increased in the purbertal and further in the postpubertal children, the level of triglyceride decreased $(\mathrm{P}<0.05)$, and the level of HDL-C increased $(\mathrm{P}<0.05)$ gradually. It indicates that as the maturation, physical activity and or training load and performance level increase the level of HDL-C and lowers the level of triglyceride among the children. The possible reason for the reduction in triglyceride and elevation in HDL-C is physical activity and exercise training [7,30,53]. However, no significant change was noted in total cholesterol and LDL-C level among the children. This might be because of low intensity and short duration of activities or improper optimization of the training load among the children. Our findings are supported by observations of other researchers in their recent studies [7,53]. Cross-sectional studies also reported an increase in HDL-C level and decrease in triglyceride level after exercise [7,53-55]. A recent study showed significant increase in HDL-C level and decrease in LDL-C level, with no change in total cholesterol and triglycerides [56]. Therefore, regular monitoring of lipids and lipoproteins profiles of young athletes is essential to optimize their health status which has direct effect on performance of the players.

\section{Conclusion}

Identification of children at early stage of their growth and development may produce elite athletes in the future. Talent identification also can be used as a counseling technique that helps to discover and explore areas of talent for particular athletes. In order to reach their goals, young children should be subjected to a series of tests reflecting anthropometric, physical and cardiorespiratory fitness which will indicate their present over all strengths and weaknesses. In addition, lipids and lipoprotein profiles of the athletes reflects their health and metabolic activities which has a positive impact on the overall performance of the athlete. Improvement in these parameters depends on level of maturation factors and / or motivation, and exposure to long term and higher intensity of training.

\section{Acknowledgment}

The authors are indebted to the University Grant Commission, New Delhi, India for financial assistance to carry out the research work. The authors sincerely and wholeheartedly acknowledge the contribution of children participated in the present study. The authors are also thankful to the under graduate students, coaches and laboratory staffs for extending their support for this study.

\section{References}

[1] Reilly, T. and Secher, N. Physiology of sports. E \& FN Spon, London, 1990, 372-425.
[2] Hoare, D.G. Predicting success in junior elite basket ball playersthe contribution of anthropometric and physiological attributes. J Sci Med Sports, 34. 391-405. 2000.

[3] Coh, M., Milanovic, D. and Embersic, D. Anthropometric characteristics of elite junior male and female javelin throwers. Coll Antropol, 26. 77-83. 2002.

[4] Katch, V.L., Mc Ardle, W.D. and Katch, F.I. Essentials of Exercise Physiology. $4^{\text {th }}$ ed. Lippincott Williams and Wilkins, Philadelphia PA, 2011.

[5] Jonathan, M., Mc Gavock, B.D., Torrance, K., Mc Guire, A., Wozny, P.D. and Lewanczuk, R.Z. Cardiorespiratory Fitness and the Risk of Overweight in Youth: The Healthy Hearts Longitudinal Study of Cardiometabolic Health. Obesity, 17. 18021807. 2009.

[6] Ortega, F.B., Ruiz, J.R., Castillo, M.J. and Sjo“stro"m, M. Physical fitness in childhood and adolescence: a powerful marker of health. Int J Obesity, 32. 1-11. 2008.

[7] Kelley, G.A. and Kelley, K.S. Impact of progressive resistance training on lipids and lipoproteins in adults: a meta-analysis of randomized controlled trials. Prev Med, 48. 9-19. 2009.

[8] Altena, T.S., Michaelson, J.L., Ball, S.D., Guilford, B.L. and Thomas, T.R. Lipoprotein subfraction changes after continuous or intermittent exercise training. Med Sci Sports Exerc, 38.367-372. 2006.

[9] Halverstadt, A., Phares, D.A., Wilund, K.R., et al. Endurance exercise training raises high-density lipoprotein cholesterol and lowers small low-density lipoprotein and very low-density lipoprotein independent of body fat phenotypes in older men and women. Metabolism, 56. 444-450. 2007.

[10] Payne, N., Gledhill, N., Katzmarzy, K.P.T., Jamnik, V.K. and Keir, P.J. Canadian musculoskeletal norms. Can J Appl Physiol, 25. 430-442. 2000.

[11] Chatterjee, S., Mandal, A. and Das, N.K. Physical and motor fitness level of Indian school going boys. J Sports Med Phys Fitness, 33. 268-277. 1993.

[12] Manna, I., Khanna, G.L. and Dhara, P.C. Age Related Changes in Selected Morphological, Physiological and Biochemical Variables of Indian Field Hockey Players. Br J Sports Med, 44 (S). i20. 2010.

[13] Manna, I., Khanna, G.L. and Dhara, P.C. Training Induced Changes on Physiological and Biochemical Variables of Young Indian Field Hockey Players. Biol Sports, 26. 33-43. 2009.

[14] Manna, I., Khanna, G.L. and Dhara, P.C. Plasma Lipids, Lipoproteins of Young Indian Athletes: A Risk Factor For Coronary Heart Disease. Australian Conference of Science and Medicine in Sport, Alice Springs, Australia. 6-9 $9^{\text {th }}$ Oct, 24, p 14. 2004.

[15] Jonson, B.L. and Nelson, J.K. Practical measurements for evaluation in physical education. Macmillan Publishing Co, London, 1986.

[16] Durnin, J.V.G.A. and Womersley, J. Body fat assessed from total body density and its estimation from skin fold thickness: measurements on 481 men and women from 16 to 72 years. Br J Nutr, 32. 77-97. 1974.

[17] Siri, W.E. The gross composition of the body. In: Tobias CA, Lawrence JH, ed. Advances in Biological and Medical Physics. Academic Press, New York, 1956, 239-280.

[18] Margaria, R., Aghemo, P. and Rovelli, E. Measurement of muscular power (anaerobic) in man. J Appl Physiol, 21. 16621664. 1996.

[19] McArdle, W.D., Katch, F.I., Pechar, G.S., Jacobson, L. and Ruck, S. Reliability and interrelationships between maximal oxygen intake, physical work capacity and step-test scores in college women. Med Sci Sports, 4. 182-186. 1972.

[20] Mustajbegovic, J., Zuskin, E., Schachter, E.N., Kern, J., LuburicMilas, M. and Pucarin, J. Respiratory findings in tobacco workers. Chest, 123. 1740-1748. 2003.

[21] Schettler, G. and Nussei, E. Maßnahmen zur Prävention der Arteriosklerose. Arb Med Soz Med Prav Med, 10. 25. 1975.

[22] Wybenga, D.R., Pileggi, V.J., Dirstine, P.H. and Di Giorgio, J. Direct manual determination of serum total cholesterol with a single stable reagent. Clin Chem, 16. 980-984. 1970.

[23] Friedewald, W.T., Levy, R.I. and Fredrickson, D.S. Estimation of the concentration of low density lipoprotein cholesterol in plasma without use of the preparative ultracentrifuge. Clin Chem, 18.499501. 1972.

[24] Dietz, W.H. Childhood weight affects adult morbidity and mortality. J Nutr, 128. 411S-414S. 1998. 
[25] Goran, M.I., Ball, G.D. and Cruz, M.L. Obesity and risk of type 2 diabetes and cardiovascular disease in children and adolescents. J Clin Endocrinol Metab, 88. 1417-1427. 2003.

[26] Guo, S.S., Chumlea, W.C., Roche, A.F. and Siervogel, R.M. Ageand maturity-related changes in body composition during adolescence into adulthood: the Fels longitudinal study. Int J Obesity, 21. 1167-1175. 1997.

[27] Katzmarzyk, P.T., Perusse, L., Malina, R.M., Bergeron, J., Despres, J.P. and Bouchard, C. Stability of indicators of the metabolic syndrome from childhood and adolescence to young adulthood: the Quebec family study. J Clin Epidemiol, 54.190195. 2001.

[28] Janssen, I., Katzmarzyk, P.T., Srinivasan, S.R., et al. Utility of childhood BMI in the prediction of adulthood disease: comparison of national and international references. Obes Res, 13. 1106-1115. 2005.

[29] Enemark-Miller, E.A., Seegmiller, J.G. and Rana, S.R. Physiological profile of women's Lacrosse players. J Strength Cond Res, 23. 39-43. 2009.

[30] Johnson, A., Doherty, P.J. and Freemont, A. Investigation of growth, development, and factors associated with injury in elite schoolboy footballers: prospective study. BMJ, 338. b490. 2009.

[31] Silvestre, R., West, C., Maresh, C.M., et al. Body composition and physical performance in men's soccer: a study of a National Collegiate Athletic Association Division I team. J Strength Cond Res, 20. 177-183. 2006.

[32] Hoff, J. Training and testing physical capacities for elite soccer players. J Sports Sci, 23. 573-582. 2005.

[33] Tahara, Y., Moji, K., Tsunawake, N. et al. Physique, body composition and maximum oxygen consumption of selected soccer players of Kunimi High School, Nagasaki, Japan. J Physiol Anthropol, 25. 291-297. 2006.

[34] Mujika, I. The influence of training characteristics and tapering on the adaptation in highly trained individuals: a review. Int J Sports Med, 19. 439-446. 1998.

[35] Coyel, E.F., Hemmert, M.K., Coggan, A.R., et al. Effect of detraining on cardiovascular responses to exercise: role of blood volume. J Appl Physiol, 60. 95-99. 1986.

[36] Reilly, T., Williams, A.M., Nevil, A. and Franks, A. A multidisciplinary approach to talent identification in soccer. $\mathrm{J}$ Sports Sci, 18. 695-702. 2000.

[37] Wilmore, J.H. and Costill, D.L. Physiology of Sport and Exercise. $3^{\text {rd }}$ ed. Human Kinetics, Champaign, IL, 2005.

[38] Bassareo, P.P., Marras, A.R., Barbanti, C. and Mercuro, G. Comparison between waist and mid-upper arm circumferences in influencing systolic blood pressure in adolescence: the SHARP (Sardinian Hypertensive Adolescent Research Programme) study. J Pediatr Neonat Individual Med, 2. 1-9. 2013.

[39] Dobbelsteyn, C.J. et al. A comparative evaluation of waist circumference, waist-to-hip ratio and body mass index as indicators of cardiovascular risk factors. The Canadian Heart Health Surveys. Int J Obes Relat Metab Disord, 25. 652-661. 2001.
[40] Yalcin, B.M., Sahin, E.M. and Yalcin, E. Which anthropometric measurement is most closely related to elevated blood pressure? Fam Pract, 22. 541-547. 2005.

[41] James, W.P.T., Mascie-Taylor, C.G.N., Norgan, N.G., Bristrian, B.R., Shetty, P. and Ferro-Luzzi, A. The value of arm circumference measurements in assessing chronic energy deficiency in Third World adults. Eur J Clin Nutr, 48. 883-894. 1994.

[42] Fukunaga, Y., Takai, Y., Yoshimoto, T., Fujita, E., Yamamoto, M. and Kanehisa, $\mathrm{H}$. Influence of maturation on anthropometry and body composition in Japanese junior high school students. J Physiol Anthropol, 32. 5. 2013.

[43] Ujević, T., Sporis, G., Milanović, Z., Pantelić, S. and Neljak, B. Differences between health-related physical fitness profiles of Croatian children in urban and rural areas. Coll Antropol, 37. 7580. 2013.

[44] Coelho-E-Silva, M.J., Vaz Ronque, E.R., Cyrino, E.S., et al. Nutritional status, biological maturation and cardiorespiratory fitness in Azorean youth aged 11-15 years. BMC Pub Health, 13. 495. 2013.

[45] Ostojic, S.M. Seasonal alterations in body composition and sprint performance of elite soccer players. J Exer Physiol on line, 6. 2427. 2003.

[46] Svensson, M. and Drust, B. Testing soccer players. J Sports Sci, 23. 601-618. 2005.

[47] Reilly, T. An Ergonomics model of the soccer training process. J Sports Sci, 6. 561-572. 2005.

[48] Ekelund, U., Poorvliet, E., Nilson, A., et al. Physical activity in relation to aerobic fitness and body fat in 14- to 15- year-old boys and girls. Eur J Appl Physiol, 85. 195-201. 2001.

[49] Astrand, P.O. and Rodhal, K. Textbook of work physiology. McGraw-Hill, New York, 1986.

[50] Rampinini, E., Impellizzeri, F.M., Castagna, C., et al. Factors influencing physiological responses to small-sided soccer games. J Sports Sci, 25. 659-666. 2007.

[51] Popadic Gacesa, J.Z., Barak, O.F. and Grujic, N.G. Maximal anaerobic power test in athletes of different sport disciplines. J Strength Cond Res, 23.751-755. 2009.

[52] Miller, T.A., Thierry-Aguilera, R., Congleton, J.J., et al. Seasonal changes in VO2max among Division 1A collegiate women soccer players. J Strength Cond Res, 21. 48-51. 2007.

[53] Heitkamp, H.C., Wegler, S., Brehme, U., et al. Effect of an 8week endurance training program on markers of antioxidant capacity in women. J Sports Med Phys Fitness, 48.113-119. 2008.

[54] Durstine, J.L., Davis, P.G., Ferguson, M.A., et al. Effects of shortduration and long-duration exercise on lipoprotein (a). Med Sci Sport Ex, 33.1511-1516. 2001.

[55] Durstine, J.L., Grandjean, P.W., Cox, C.A. and Thompson, P.D. Lipids, lipoproteins, and exercise. J Cardiopulm Rehabil, 22. 385398. 2002.

[56] Degoutte, F., Jouanel, P., Begue, R.J., et al. Food restriction, performance, biochemical, psychological, and endocrine changes in judo athletes. Int J Sports Med, 27. 9-18. 2006. 